\title{
Four quark operators in maximally twisted Wilson LQCD
}

\section{Roberto Frezzotti ${ }^{* \dagger}$}

INFN, Sezione di Milano and Università di Milano "Bicocca”, P.zza della Scienza 3 - 20126

Milano (Italy)

E-mail: Roberto.Frezzotti@mib.infn.it

\section{Giancarlo Rossi}

Università di Roma “Tor Vergata”, Dipartimento di Fisica, Via della Ricerca Scientifica - 00133

Roma (Italy)

\section{E-mail: Giancarlo.Rossi@roma2.infn.it}

\begin{abstract}
We discuss how the peculiar properties of maximally twisted Wilson fermions can be exploited to set up a consistent LQCD computational scheme in which the CP-conserving matrix elements of the $\Delta S=1,2$ effective weak Hamiltonian can be evaluated without mixing with wrong-chirality and/or -parity operators. The proposed lattice framework guarantees automatic $\mathrm{O}(a)$ improvement and positive determinant also for pairs of non-degenerate quarks.
\end{abstract}

XXIIIrd International Symposium on Lattice Field Theory

25-30 July 2005

Trinity College, Dublin, Ireland

\footnotetext{
* Speaker.

†From September 1st, 2005, new affiliation and address: Università di Roma “Tor Vergata”, Dipartimento di Fisica, Via della Ricerca Scientifica - 00133 Roma (Italy); e-mail: Roberto.Frezzotti@ roma2.infn.it .
} 


\section{Introduction and main results}

We outline here a rather general strategy [1] to compute weak matrix elements -and more generally operator matrix elements among hadronic states- using maximally twisted Wilson fermions in such a way that wrong-chirality and -parity mixings, as well as $\mathrm{O}(a)$ discretization errors, are avoided and positivity of the fermionic determinant is guaranteed ${ }^{1}$. Our strategy relies on

- the existence of renormalizable Euclidean lattice gauge models including "sea" quarks as well as possibly replicated "valence" quarks (and corresponding ghost fields), from which one can extract, among others, operator matrix elements that in the continuum limit coincide with those of the effective weak Hamiltonian in QCD;

- the possibility of choosing the (sign of the) Wilson parameters for the various replica of (maximally twisted, single flavour) "valence" quark fields, as well as the way they enter the relevant quark operators, so as to avoid wrong-chirality or -parity mixings.

The latter property has so far [1] been established only for the CP-conserving $\Delta S=1,2$ effective weak Hamiltonian operators (see discussion below) but is likely to be more general. We also assume in the following that the "infrared" problems in the lattice computation of matrix elements for multiparticle states [3] have been solved in some way, e.g. by the methods of Refs. [ $€$ ].

We focus here on matrix elements of four quark operators. The evaluation of matrix elements of two quark operators without unnecessary mixings and $\mathrm{O}(a)$ cutoff effects is in several cases feasible within the framework of plain twisted mass Lattice QCD (tmLQCD) [5] (even in the case of mass non-degenerate quark pairs [6]) and will not be discussed here ${ }^{2}$. For recent reviews on tmLQCD see Refs. [8]. An extension of our approach to the computation of $B_{B}$ in the static heavy quark limit has been proposed in Ref. [饮.

Other methods for the evaluation of specific four fermion matrix elements using twisted Wilson fermions with no (or reduced) wrong-chirality and -parity mixings are possible and have in fact been developed for the cases of $B_{K}$ [5, 10], the $\Delta I=1 / 2$ rule [11] and $B_{B}$ in the static heavy quark limit [12]. For recent reviews on weak matrix elements in LQCD see e.g. Refs. [13].

\section{2. $4 \mathrm{sNv}$ Euclidean lattice models}

Let us start by specifying the lattice formulation of a generic (Euclidean SU(3)) gauge model with "sea" quarks as well as $N$ flavours of "valence" quarks and corresponding ghosts we alluded to above. For definiteness we specialize to the realistic case of four non-degenerate physical quark flavours $(u, d, s$ and $c)$. The lattice action takes thus the form

$$
S_{4 S N v}=S_{g}[U]+S_{\mathrm{tm}}^{(\pi / 2)}\left[\psi_{\ell}, \bar{\psi}_{\ell}, U\right]+S_{\mathrm{tm}}^{(\pi / 2)}\left[\psi_{h}, \bar{\psi}_{h}, U\right]+\sum_{f=1}^{N}\left[S_{\mathrm{OS}}^{(\pi / 2)}\left[q_{f}, \bar{q}_{f}, U\right]+S_{g h}^{(\pi / 2)}\left[\phi_{f}, U\right]\right]
$$

\footnotetext{
${ }^{1}$ We do not discuss here the various possible ways of performing the (non-perturbative) renormalization of the matrix elements of interest. However, if one wishes to employ a Schrödinger functional scheme without reintroducing $\mathrm{O}(a)$ bulk discretization errors, modifications of the usual setup are needed: see Refs. [2] for two different proposals.

${ }^{2}$ We only recall a recently proposed application of tmLQCD to the evaluation of $V_{u s}$, 77 .
} 
where ${ }^{3} S_{g}[U]$ is a suitable pure gauge action ${ }^{4}$,

$$
S_{\mathrm{tm}}^{(\pi / 2)}\left[\psi_{p}, \bar{\psi}_{p}, U\right]=a^{4} \sum_{x} \bar{\psi}_{p}(x)\left[\gamma \cdot \widetilde{\nabla}-i \gamma_{5} \tau_{1} W_{\mathrm{cr}}\left(r_{p}\right)+m_{p}-\varepsilon_{p} \tau_{3}\right] \psi_{p}(x), \quad p=\ell, h
$$

is the action for a pair of "sea" quarks $\left(\psi_{\ell}=(u, d), \psi_{h}=(s, c)\right.$ and typically $\left.\left|r_{p}\right|=1\right)$ with

$$
W_{\mathrm{cr}}(r)=-a \frac{r}{2} \sum_{\mu} \nabla_{\mu}^{\star} \nabla_{\mu}+M_{\mathrm{cr}}(r)
$$

while

$$
\begin{aligned}
& S_{\mathrm{OS}}^{(\pi / 2)}\left[q_{f}, \bar{q}_{f}, U\right]=a^{4} \sum_{x} \bar{q}_{f}(x)\left[\gamma \cdot \widetilde{\nabla}-i \gamma_{5} W_{\mathrm{cr}}\left(r_{f}\right)+m_{f}\right] q_{f}(x), \\
& S_{g h}^{(\pi / 2)}\left[\phi_{f}, U\right]=a^{4} \sum_{x} \phi_{f}^{\dagger}(x) \operatorname{sign}\left(m_{f}\right)\left[\gamma \cdot \widetilde{\nabla}-i \gamma_{5} W_{\mathrm{cr}}\left(r_{f}\right)+m_{f}\right] \phi_{f}(x)
\end{aligned}
$$

represent the action for a single flavour, $q_{f}$, Österwalder-Seiler (OS) fermion [15] and that for the corresponding ghost field, $\phi_{f}$, respectively. The latter is a c-number, spin $1 / 2$ lattice field belonging to the fundamental representation of the gauge group SU(3). Integration over $\phi_{f}$ is convergent and yields an inverse single-flavour determinant that cancels the corresponding determinant arising from "integration" over the Grassmann fields $q_{f}$ and $\bar{q}_{f}$.

Renormalizability of the lattice $4 \mathrm{sNv}$ model with action (2.1) follows from standard power counting and symmetry [1], 16] arguments: for more details, such as the precise definition of the critical masses appearing in eqs. (2.2) to (2.5), see Ref. [1]. Here we only recall that valence flavour is obviously conserved and all valence (current) quark masses renormalize multiplicatively

$$
\hat{m}_{f}=Z_{m}\left(r_{f}\right) m_{f},
$$

while the renormalized sea (current) quark masses take the form

$$
\begin{array}{cl}
m_{u_{\mathrm{sea}}} \equiv m_{\ell}^{-}=Z_{P}^{-1}\left(r_{\ell}\right) m_{\ell}-Z_{S}^{-1}\left(r_{\ell}\right) \varepsilon_{\ell}, & m_{d_{\mathrm{sea}}} \equiv m_{\ell}^{+}=Z_{P}^{-1}\left(r_{\ell}\right) m_{\ell}+Z_{S}^{-1}\left(r_{\ell}\right) \varepsilon_{\ell} \\
m_{s_{\mathrm{sea}}} \equiv m_{h}^{-}=Z_{P}^{-1}\left(r_{h}\right) m_{h}-Z_{S}^{-1}\left(r_{h}\right) \varepsilon_{h}, & m_{c_{\mathrm{sea}}} \equiv m_{h}^{+}=Z_{P}^{-1}\left(r_{h}\right) m_{h}+Z_{S}^{-1}\left(r_{h}\right) \varepsilon_{h} .
\end{array}
$$

One can show that in a mass independent scheme all these (sea and valence) renormalized quark masses have a common scale dependence.

In the following we will restrict attention to vacuum expectation values of multilocal operators made out exclusively of valence quark fields. Automatic $\mathrm{O}(a)$ improvement (and absence of all discretization errors coming with odd powers of the lattice spacing) of parity-even correlators and derived quantities follows from the invariance of the lattice $4 \mathrm{sNv}$ model under $\mathscr{P} \times \mathscr{D}_{d} \times(M \rightarrow$ $-M)$, with $M$ an array made out of the $4+N$ bare quark mass parameters. The precise definitions of $\mathscr{P}$ (the physical parity operation) and $\mathscr{D}_{d}$ can be found in Ref. [1]. Since the effect of $\mathscr{D}_{d} \times(M \rightarrow$ $-M)$ on a generic operator of naive dimension $d$ is to multiply it by $(-1)^{d}$, besides reflecting all its spacetime arguments, a simple argument for automatic $\mathrm{O}(a)$ improvement can be made that is closely analogous to the argument given in Ref. [17] for plain tmLQCD ${ }^{5}$.

\footnotetext{
${ }^{3}$ For undefined notations here and in the following we refer the reader to Ref. [1].

${ }^{4}$ In unquenched computations a clever choice of $S_{g}[U]$ can be important for the phase structure of the lattice model and thus crucial to be able to perform simulations at maximal twist with no metastability problems (see Refs. [14, 8).

${ }^{5}$ A slightly different argument, which employs the $r$-parity properties of the critical masses, was given in Ref. [i卬].
} 


\section{3. $B_{K}$ and $\Delta I=1 / 2$ rule: prescription for bare matrix elements}

The general strategy of our approach is as follows. Let $\langle\beta|\mathscr{O}| \alpha\rangle$ be the matrix element of the local operator $\mathscr{O}$ between hadronic states $|\alpha\rangle$ and $|\beta\rangle$-evaluated in QCD with four flavours (qcd4)we are interested in. Usually $\langle\beta|\mathscr{O}| \alpha\rangle$ is extracted from a suitable qcd4 correlator, but, as argued below, it can equally well be extracted, up to discretization errors, from correlators of the form

$$
C_{\Phi_{\beta} \mathscr{Q} \Phi_{\alpha}}^{(4 s N v)}(x, y)=\left\langle\Phi_{\beta}\left(x_{1}, x_{2}, \ldots\right) \mathscr{Q}(0) \Phi_{\alpha}\left(y_{1}, y_{2}, \ldots\right)\right\rangle^{(4 s N v)},
$$

evaluated in an appropriate $4 \mathrm{sNv}$ lattice model where $\mathscr{Q}, \Phi_{\alpha}$ and $\Phi_{\beta}$ are suitable operators whose form is determined by $\mathscr{O}$ and the states $|\alpha\rangle$ and $|\beta\rangle$, respectively. Our approach is illustrated in [1], where we discuss in some detail a few cases of phenomenological relevance. The main points of that discussion are summarized below. One can check that in all these cases the number of valence (Mtm-Wilson) quark propagators to be computed is only 1.5 times larger than usually.

\section{1 $K^{0}-\bar{K}^{0}$ mixing amplitude}

The target qcd4 matrix element (renormalized at the scale $\mu$ ), namely $\left\langle\bar{K}^{0}\left|\hat{\mathcal{O}}_{V V+A A}^{\Delta S=2}(\mu)\right| K^{0}\right\rangle \equiv$ $\frac{16}{3} M_{K}^{2} F_{K}^{2} \hat{B}_{K}(\mu)$, is usually extracted from the correlator $\left(x_{0}>0, y_{0}<0\right)$

$$
C_{K \mathscr{O} K}^{(\mathrm{qcd} 4)}(x, y)=\left\langle\left(\bar{d} \gamma_{5} s\right)(x) \widehat{\mathscr{O}}_{V V+A A}^{\Delta S=2}(0)\left(\bar{d} \gamma_{5} s\right)(y)\right\rangle
$$

with the bare expression of $\widehat{\mathscr{O}}_{V V+A A}^{\Delta S=2}$ given by $\mathscr{O}_{V V+A A}^{\Delta S=2}=\left(\bar{s} \gamma_{\mu} d\right)\left(\bar{s} \gamma_{\mu} d\right)+\left(\bar{s} \gamma_{\mu} \gamma_{5} d\right)\left(\bar{s} \gamma_{\mu} \gamma_{5} d\right)$. We propose to consider a $4 \mathrm{~s} 6 \mathrm{v}$ model, with valence quarks $u, d, d^{\prime}, s, s^{\prime}, c$, and extract the bare matrix element

$$
\left\langle\bar{K}^{\prime 0}\left|\mathscr{Q}_{V V+A A}^{\Delta S=2}\right| K^{0}\right\rangle=\frac{16}{3} M_{K^{\prime}} F_{K^{\prime}} M_{K} F_{K} B_{K}^{\text {bare }} .
$$

from a correlator of the form (3.1) with $\Phi_{\beta}=\bar{d}^{\prime} \gamma_{5} s^{\prime}, \Phi_{\alpha}=\bar{d} \gamma_{5} s$,

$$
\mathscr{Q}_{V V+A A}^{\Delta S=2}=2\left[\left(\bar{s} \gamma_{\mu} d\right)\left(\bar{s}^{\prime} \gamma_{\mu} d^{\prime}\right)+\left(\bar{s} \gamma_{\mu} \gamma_{5} d\right)\left(\bar{s}^{\prime} \gamma_{\mu} \gamma_{5} d^{\prime}\right)+\left(\bar{s} \gamma_{\mu} d^{\prime}\right)\left(\bar{s}^{\prime} \gamma_{\mu} d\right)+\left(\bar{s} \gamma_{\mu} \gamma_{5} d^{\prime}\right)\left(\bar{s}^{\prime} \gamma_{\mu} \gamma_{5} d\right)\right]
$$

and the lattice regularization fixed by ${ }^{6} r_{d}=r_{d^{\prime}}=r_{s}=-r_{s^{\prime}}$. Direct symmetry arguments thus imply [1] that $\mathscr{Q}_{V V+A A}^{\Delta S=2}$, eq. (3.4), renormalizes multiplicatively.

\section{2 $K \rightarrow \pi \pi$ amplitudes}

The amplitudes for weak $K \rightarrow \pi \pi$ decays in the $\Delta I=1 / 2,3 / 2$ channels can be expressed in terms of Wilson coefficients and the (renormalized) matrix elements $\left\langle\pi^{+} \pi^{-}\left|\widehat{\mathscr{O}}_{V A}^{ \pm}+A V(\mu)\right| K^{0}\right\rangle$, $\left\langle\pi^{0} \pi^{0}\left|\widehat{\mathscr{O}}_{V A+A V}^{ \pm}(\mu)\right| K^{0}\right\rangle$ with the bare expression of $\widehat{\mathscr{O}}_{V A+A V}^{ \pm}(\mu)$ given by

$$
\begin{aligned}
\mathscr{O}_{V A+A V}^{ \pm}= & \frac{1}{2}\left[\left(\bar{s} \gamma_{\mu} u\right)\left(\bar{u} \gamma_{\mu} \gamma_{5} d\right) \pm\left(\bar{s} \gamma_{\mu} d\right)\left(\bar{u} \gamma_{\mu} \gamma_{5} u\right)\right]-\frac{1}{2}[u \leftrightarrow c]+ \\
& +\frac{1}{2}\left[\left(\bar{s} \gamma_{\mu} \gamma_{5} u\right)\left(\bar{u} \gamma_{\mu} d\right) \pm\left(\bar{s} \gamma_{\mu} \gamma_{5} d\right)\left(\bar{u} \gamma_{\mu} u\right)\right]-\frac{1}{2}[u \leftrightarrow c] .
\end{aligned}
$$

\footnotetext{
${ }^{6}$ The $u$ and $c$ valence flavours do not enter explicitly here, their regularization can thus be left unspecified.
} 
We propose to consider a 4s10v model, with valence quarks $u, u^{\prime}, u^{\prime \prime}, u^{\prime \prime \prime}, d, s, c, c^{\prime}, c^{\prime \prime}, c^{\prime \prime \prime}$, and extract the bare matrix elements ${ }^{7}\left\langle\pi^{+} \pi^{-}\left|\mathscr{Q}_{V A+A V}^{ \pm}\right| K^{0}\right\rangle$ and $\left\langle\pi^{0} \pi^{0}\left|\mathscr{Q}_{V A+A V}^{ \pm}\right| K^{0}\right\rangle$ from (connected) correlators of the form (3.1), namely

$$
\begin{aligned}
C_{ \pm, K^{0} \pi^{+} \pi^{-}}^{(4 s 10 v)}\left(x_{1}, x_{2}, y\right) & =\left\langle\Phi_{\pi^{+} \pi^{-}}\left(x_{1}, x_{2}\right) \mathscr{Q}_{V A+A V}^{ \pm}(0) \Phi_{K^{0}}^{\dagger}(y)\right\rangle_{\mathrm{conn}}^{(4 s 10 v)} \\
C_{ \pm, K^{0} \pi^{0} \pi^{0}}^{(4 s 10 v)}\left(x_{1}, x_{2}, y\right) & =\left\langle\Phi_{\pi^{0} \pi^{0}}\left(x_{1}, x_{2}\right) \mathscr{Q}_{V A+A V}^{ \pm}(0) \Phi_{K^{0}}^{\dagger}(y)\right\rangle_{\mathrm{conn}}^{(4 s 10 v)}
\end{aligned}
$$

The meson interpolating fields $\Phi_{K^{0}}^{\dagger}, \Phi_{\pi^{+} \pi^{-}}$and $\Phi_{\pi^{0} \pi^{0}}$ are made out of valence $u, d$ and $s$ quarks only and the four fermion operator takes the form (see eq. (3.5)

$$
\mathscr{Q}_{V A+A V}^{ \pm}=\mathscr{O}_{V A+A V}^{ \pm[0]}+\mathscr{O}_{V A+A V}^{ \pm[1]}-\frac{1}{2} \mathscr{O}_{V A+A V}^{ \pm[2]}-\frac{1}{2} \mathscr{O}_{V A+A V}^{ \pm[3]}
$$

with $\mathscr{O}_{V A+A V}^{ \pm[0]}$ involving $u$ and $c$ valence quark fields, $\mathscr{O}_{V A+A V}^{ \pm[1]}$ involving $u^{\prime}$ and $c^{\prime}$ and so on. The key point to avoid wrong-chirality or -parity mixings of $\mathscr{Q}_{V A+A V}^{ \pm}$is to fix the lattice regularization by

$$
r_{s}=r_{d}=r_{u}=-r_{u^{\prime}}=r_{u^{\prime \prime}}=-r_{u^{\prime \prime \prime}}=r_{c}=-r_{c^{\prime}}=r_{c^{\prime \prime}}=-r_{c^{\prime \prime \prime}} .
$$

\section{3 $K \rightarrow \pi$ amplitudes}

Since in the chiral limit $K \rightarrow \pi \pi$ amplitudes can be related to $K \rightarrow \pi$ and $K \rightarrow$ vacuum amplitudes, valuable information on the $\Delta I=1 / 2$ rule can also be obtained by evaluating in qcd 4 -for several "small" external momenta and quark mass values- the (simpler) matrix elements $\left\langle\pi^{+}(p)\left|\widehat{\mathscr{O}}_{V V+A A}^{ \pm}\right| K^{+}(q)\right\rangle$ and $\left\langle\pi^{0}(p)\left|\widehat{\mathscr{O}}_{V V+A A}^{ \pm}\right| K^{0}(q)\right\rangle$, with the bare four quark operator given by

$$
\begin{aligned}
\mathscr{O}_{V V+A A}^{ \pm}= & \frac{1}{2}\left[\left(\bar{s} \gamma_{\mu} u\right)\left(\bar{u} \gamma_{\mu} d\right) \pm\left(\bar{s} \gamma_{\mu} d\right)\left(\bar{u} \gamma_{\mu} u\right)\right]-\frac{1}{2}[u \leftrightarrow c]+ \\
& +\frac{1}{2}\left[\left(\bar{s} \gamma_{\mu} \gamma_{5} u\right)\left(\bar{u} \gamma_{\mu} \gamma_{5} d\right) \pm\left(\bar{s} \gamma_{\mu} \gamma_{5} d\right)\left(\bar{u} \gamma_{\mu} \gamma_{5} u\right)\right]-\frac{1}{2}[u \leftrightarrow c]
\end{aligned}
$$

In analogy with the case of sect. (3.2), one can extract the bare matrix elements $\left\langle\pi^{+} \pi^{-}\left|\mathscr{Q}_{V A+A V}^{ \pm}\right| K^{0}\right\rangle$ and $\left\langle\pi^{0} \pi^{0}\left|\mathscr{Q}_{V A+A V}^{ \pm}\right| K^{0}\right\rangle$ in a 4s10v model from (connected) correlators of the form (3.1), namely

$$
C_{ \pm, K \pi}^{(4 s 10 v)}(x, y)=\left\langle\Phi_{\pi}(x) \mathscr{Q}_{V V+A A}^{ \pm}(0) \Phi_{K}^{\dagger}(y)\right\rangle_{\mathrm{conn}}^{(4 s 10 v)},
$$

where $K$ and $\pi$ is either $K^{0}$ and $\pi^{0}$ or $K^{+}$and $\pi^{+}$, the interpolating $K$ and $\pi$ fields involve only the $u, d$ and $s$ valence quarks and, in analogy to eq. (3.8), $\mathscr{Q}_{V V+A A}^{ \pm}=\mathscr{O}_{V V+A A}^{ \pm[0]}+\mathscr{O}_{V V+A A}^{ \pm[1]}-\frac{1}{2} \mathscr{O}_{V V+A A}^{ \pm[2]}-$ $\frac{1}{2} \mathscr{O}_{V V+A A}^{ \pm[3]}$. To have no unwanted mixings the lattice regularization can now be taken such that

$$
-r_{s}=r_{d}=r_{u}=-r_{u^{\prime}}=r_{u^{\prime \prime}}=-r_{u^{\prime \prime \prime}}=r_{c}=-r_{c^{\prime}}=r_{c^{\prime \prime}}=-r_{c^{\prime \prime \prime}} .
$$

\section{Contact with QCD correlators and matrix elements}

Taking the example of the $K^{0}-\bar{K}^{0}$ mixing amplitude, we briefly explain the relation between the correlators -and derived matrix elements- in the chosen $4 \mathrm{~s} 6 \mathrm{v}$ model and those of qcd $4{ }^{8}$.

\footnotetext{
${ }^{7}$ As argued below, the renormalized counterparts of these matrix elements yield lattice estimates of the correspondingly renormalized qcd4 target matrix elements, $\left\langle\pi^{+} \pi^{-}\left|\widehat{\mathscr{O}}_{V A+A V}^{ \pm}(\mu)\right| K^{0}\right\rangle$ and $\left\langle\pi^{0} \pi^{0}\left|\widehat{\mathscr{O}}_{V A+A V}^{ \pm}(\mu)\right| K^{0}\right\rangle$.

${ }^{8}$ For more details and the analogous arguments relevant for the amplitudes discussed in sect. 3.2 3.3 see Ref. [1].
} 
The crucial point is that the $4 \mathrm{~s} 6 \mathrm{v}$ correlator (3.1), with $\Phi_{\beta}=\bar{d}^{\prime} \gamma_{5} s^{\prime}, \Phi_{\alpha}=\bar{d} \gamma_{5} s$ and the operator $\mathscr{Q}_{V V+A A}^{\Delta S=2}$ specified in eq. (3.4), coincides with the qcd4 correlator (3.2), if (all) the quarks of the same physical flavour are regularized in the same way in the two theories. The equality immediately follows from the identity of Wick contractions. In particular it implies that "corresponding" local operators, such as $\mathscr{Q}_{V V+A A}^{\Delta S=2}$ in $4 \mathrm{~s} 6 \mathrm{v}$ and $\mathscr{O}_{V V+A A}^{\Delta S=2}$ in qcd4, admit the same renormalization constant (and thus equal non-perturbative anomalous dimension). Moreover renormalized (current) quark masses of the same flavour are taken equal in $4 \mathrm{~s} 6 \mathrm{v}$ and qcd4. Under these conditions, the equality of $C_{\Phi_{\beta} \mathscr{Q} \Phi_{\alpha}}^{(4 s 6 v)}(x, y)$ and $C_{\Phi_{\beta} \mathscr{O} \Phi_{\alpha}}^{(q c d 4)}(x, y)$ carries over to the continuum limit, implying equality (as $a \rightarrow 0$ ) of the renormalized matrix elements that can be extracted from them, including $\hat{B}_{K}$.

The advantage of working with the $4 \mathrm{~s} 6 \mathrm{v}$ model rather than with qcd 4 is the greater flexibility in the choice of its lattice regularization, which results from the possibility of giving different values to the Wilson parameters of different replica of the same physical flavour. This flexibility has been exploited in sect. 3.1 by taking $r_{d}=r_{d^{\prime}}=r_{s}=-r_{s^{\prime}}$ and is the key to obtain the discrete symmetries that ensure [1] multiplicative renormalizability (i.e. no mixing) of the operator $\mathscr{Q}_{V V+A A}^{\Delta S=2}$.

\section{Acknowledgements}

We thank the LOC of Lattice 2005 for the very stimulating atmosphere of the conference.

\section{References}

[1] R. Frezzotti and G.C. Rossi, JHEP 0410 (2004) 070 (hep-lat/0407002).

[2] R. Frezzotti and G.C Rossi, hep-lat/0507030. S. Sint, these Proceedings.

[3] L. Maiani and M. Testa, Phys. Lett. B 245 (1990) 585.

[4] L. Lellouch and M. Lüscher, Commun. Math. Phys. 219 (2001) 31 (hep-lat/0003023). C.J.D. Lin, G. Martinelli, C.T. Sachrajda and M. Testa, Nucl. Phys. B 619 (2001) 467 (hep-lat/0104006).

[5] R. Frezzotti, P.A. Grassi, S. Sint and P. Weisz [Alpha collaboration], JHEP 0108 (2001) 058; Nucl. Phys. Proc. Suppl. 83 (2000) 941.

[6] R. Frezzotti and G.C. Rossi, Nucl. Phys. Proc. Suppl. 128 (2004) 193 (hep-lat/0311008).

[7] G. Isidori, G. Martinelli and P. Turchetti, hep-lat/0506026.

[8] A. Shindler, these Proceedings; R. Frezzotti, Nucl. Phys. Proc. Suppl. 140, 134 (2005).

[9] M. Della Morte, Nucl. Phys. Proc. Suppl. 140, 458 (2005) (hep-lat/0409012); talk Dec. 2004, http://linde.physik.hu-berlin.de/talksseminars.html.

[10] P. Dimopoulos et al., Nucl. Phys. Proc. Suppl. 140, 362 (2005) (hep-lat/0409026) and Refs. therein.

[11] C. Pena, S. Sint and A. Vladikas, JHEP 0409, 069 (2004) (hep-lat/0405028).

[12] F. Palombi, M. Papinutto, C. Pena and H. Wittig [ALPHA Collaboration], hep-lat/0509008.

[13] C. Dawson, these Proceedings; M. Okamoto, these Proceedings.

[14] F. Farchioni et al., Eur. Phys. J. C 42, 73 (2005) (hep-lat/0410031).

[15] K. Österwalder and E. Seiler, Annals Phys. 110 (1978) 440.

[16] S.R. Sharpe and N. Shoresh, Phys. Rev. D 64, 114510 (2001) (hep-lat/0108003).

[17] R. Frezzotti, G. Martinelli, M. Papinutto and G.C. Rossi, hep-lat/0503034. 